\title{
PLASTIC BUCKLING BEHAVIOR OF A THIN-PLATE STRUCTURE BY LATERAL IMPACT LOADING
}

\author{
Kyung-Ho Yoon, Heung-Seok Kang, Hyung-Kyu Kim, and Kee-Nam Song \\ Korea Atomic Energy Research Institute,
}

\section{INTRODUCTION}

Inserted thin plates compose the grid structure and then laser welded at the cross-points and the connection points of the inner/outer plates. The boundary condition of this structure was that one surface was simply supported, and the other surface was imposed for the impact load in a lateral direction. The critical impact velocity and force were important scales for evaluating the integrity of the structure under the above boundary condition.

In this work, FE analysis is established for predicting the plastic buckling behavior of the multi-cell grid structure under the lateral impact loading. The main purpose of this was to develop the finite element analysis model for predicting the impact test behavior. In previous work, it was mainly dependent on the direct impact test; however, it needed recursively testing for the change of the boundary condition and geometry of the structure. The current considered FE model was a multi-point constraints model case, in which all the slit edges were actually separated with translational and rotational degree of freedom.

The FE model was created with 4-node shell element, rigid and mass element using I-DEAS [4]. ABAQUS/explicit commercial code was used for solver. The FE analysis procedure was supposed, an impact hammer was modeled as the rigid element, which had equivalent mass with their hammer. The external impact load was modeled on the initial impact velocity at the reference node of the upper rigid surface, which was located in the centre. The FE analyses results were investigated by impact acceleration, velocity, force and deformed shape.

\section{CONCLUSIONS}

Finite element analyses were executed for evaluating the dynamic buckling behaviour of the grid structure. From the facts described above, we concluded that the certain method for investigating the dynamic soundness of the grid structure was proposed to examine the maximum equivalent strain of it. And then the critical parameters of the dynamic buckling under the lateral impact loading were depends upon the perimeter strap of a grid. It was that the buckling behavior was likely to occur at the perimeter strap. In addition to these, there were no relate to the friction factor between two contact surfaces 